\title{
Caracterização de bots no Twitter durante as Eleições Presidenciais no Brasil em 2018
}

\author{
Gabriel P. Nobre ${ }^{1}$, Jussara M. Almeida ${ }^{1}$, Carlos H. G. Ferreira ${ }^{1,2}$ \\ ${ }^{1}$ Universidade Federal de Minas Gerais \\ ${ }^{2}$ Universidade Federal de Ouro Preto \\ \{gnobre, jussara, chgferreira\}@dcc.ufmg.br
}

\begin{abstract}
In 2018 occurred the Brazil's presidential elections and it was widely spread on the media that bots were used in social media to share fake news and increase online support. That given, the goal on this paper is to characterize bot's behavior in Twitter during the electoral campaign. To do so, we identified 2,000 bot users, calculated behavior metrics and compared them with common users. After that, we were able to estimate their influence on the social network by quantifying influence metrics and the results suggested that they indeed affected the online discussions.
\end{abstract}

Resumo. Em 2018 ocorreram as Eleições Presidenciais no Brasil e foi frequentemente divulgado pela mídia que bots foram utilizados nas redes sociais para compartilhar fake news e aumentar o engajamento de candidatos. Com isso, o objetivo deste trabalho é caracterizar o comportamento desses usuários no Twitter durante as campanhas eleitorais. Para tanto, foram identificados mais de 2000 usuários bots, com métricas de atividade calculadas e comparadas com usuários comuns. Enfim, a influência de tais usuários foi quantificada e os resultados indicam que os bots afetaram as discussões online.

\section{Introdução}

O sistema de interações humanas nas redes sociais tem frequentemente instigado estudos sobre como o compartilhamento de conteúdo online afeta e pode ser afetado por eventos no mundo real. Nesse contexto, torna-se necessário discutir a contribuição dessas mídias em acontecimentos sociopolíticos, como eleições, guerras civis, eventos esportivos e acidentes naturais.

Trabalhos recentes têm se dedicado a estudar a interação de contas automatizadas (usuários bots) em diferentes redes e contextos sociais. Eles estudam, por exemplo, detecção de bots [Abokhodair et al. 2015, Ferrara et al. 2014, Rizoiu et al. 2018, Dong and Liu 2018], como é o conteúdo compartilhado por bots [Caetano et al. 2017, Caetano et al. 2018], a interferência dos bots na dinâmica de compartilhamento de informações [Ratkiewicz et al. 2011, Rizoiu et al. 2018], bem como em eventos externos a Internet [Ferrara et al. 2014].

Dada a possibilidade de que contas automatizadas podem interferir em eventos sociais, surgiram trabalhos que estudam como esse fenômeno pode acontecer [Messias et al. 2013, Bence Kollanyi and Woolley 2016]. Por exemplo, a interferência de bots que pode ter acontecido durante as eleições presidenciais no Estados Unidos da 
América em 2016 tem sido estudada recentemente [Bessi and Ferrara 2016] e um número de trabalhos concorda que bots afetam discussões online [Ferrara et al. 2014].

Com indícios de que atividades de usuários automatizados podem interferir em eventos sociais, o objetivo deste trabalho é estudar o comportamento de bots no Twitter durante as Eleições Presidenciais no Brasil em 2018. Esse evento político esteve em evidência na mídia devido à detecção de compartilhamento de fake news e da utilização de bots para propagá-las em plataformas como Twitter, WhatsApp e Facebook [Cristina Tardáguila and Ortellado 2018, Long 2018, Owen 2018]. Sendo assim, neste trabalho estudamos características de comportamento de bots e as comparamos com usuários comuns, além de calcular e analisar suas métricas de influência no Twitter.

Para guiar a realização do objetivo deste trabalho, foram definidas duas Questões de Pesquisa (QP):

- QP1: Usuários bots estavam presentes no Twitter em meio a discussões sobre as Eleições Presidenciais no Brasil em 2018? Se sim, como se compara a atividade de usuários bots com usuários comuns?

- QP2: Se sim, estes usuários bots influenciaram usuários comuns?

Para responder as QP's surgem três desafios. O primeiro desafio é coletar uma base de dados abrangente que possibilite as análises desejadas. Para tanto, coletamos cerca de 40 milhões de tweets que continham pelo menos o nome de um dos quatorze candidatos a presidência. O segundo é detectar bots na base de dados para comparar as métricas de atividade com usuários comuns. Com isso, classificamos 100 mil usuários da base coletada e os separamos em grupos de níveis de automação para, então, realizar as comparações de comportamento pela análise do resultado do cálculo de 18 métricas de atividade. O terceiro é relacionado ao cálculo de métricas de influência no Twitter nesse contexto. Calculamos duas diferentes métricas de influência inspiradas dos trabalhos relacionados e avaliamos se bots foram hábeis em influenciar usuários comuns. Para superar tais desafios, foi realizada uma pesquisa de trabalhos relacionados para identificar as técnicas a serem utilizadas.

As seções seguintes deste trabalho estão organizadas da seguinte forma: A Seção 2 discute trabalhos relacionados. Na Seção 3, apresentamos a metodologia utilizada. A Seção 4 detalhas os resultados experimentais obtidos. A Seção 5 discute os resultados obtidos relacionando-os com as QP's. E por fim, a Seção 6 conclui este estudo.

\section{Trabalhos relacionados}

Os trabalhos relacionados pesquisados podem se dividir em duas seções. A primeira se refere a trabalhos de identificação e caracterização de usuários bots no Twitter, bem como a influência desses usuários em eventos externos ao mundo online. A segunda seção reúne trabalhos que estudam métricas de influência de usuários no Twitter.

\subsection{Identificação e caracterização de bots}

Nesta seção de trabalhos relacionados relacionamos as pesquisas que estudaram mecanismos de detectar bots e apresentaram uma metodologia para caracterizar o comportamento de tais usuários. 
A tarefa de identificar bots em uma rede social diversa como a de usuários do Twitter pode ser realizada por meio de diferentes abordagens. Com o objetivo de obter uma rápida detecção, [Howard and Kollanyi 2016] utilizou a métrica de número de tweets por dia de cada usuário e classificou como bot aqueles que passaram um limite mínimo. Em outro trabalho [Abokhodair et al. 2015], pesquisadores conseguiram detectar uma rede de bots manualmente. Já a pesquisa apresentada em [Dickerson et al. 2014] a detecção ocorreu com o desenvolvimento de uma ferramenta, denominada SentiBot, que se utiliza de métricas de tweets como sintaxe e semântica e, também, de característica do perfil do conta e imersão do usuário na rede do Twitter. Em outros trabalhos [Rizoiu et al. 2018, Caetano et al. 2018, Rizoiu et al. 2018], os pesquisadores utilizaram a Botometer API.

A Botometer API [Dong and Liu 2018] provê uma maneira de fácil utilização para classificar um usuário do Twitter como um usuário comum ou bot. Segundo os autores, são utilizadas mais de 1000 características das contas dos usuários em um modelo criado com o algoritmo Floresta Aleatória e retornando o CAP (do Inglês Complete Automation Probability, Probabilidade de Automação Completa) sendo necessário como entrada apenas o identificador de cada um. O CAP é um valor que varia de 0 a 1 e se refere a probabilidade de um usuário ser completamente automatizado, um bot. Os autores recomendam que usuários com CAP maior que 0.5 sejam classificados como bots.

O objetivo de muitos trabalhos é identificar bots e estudar como eles podem influenciar eventos externos à rede do Twitter. Tais trabalhos pesquisam esse assunto em diferentes contextos, como eventos sociais, políticos, eleições e guerras civis.

A influência de bots no Twitter é tópico de interesse em diferentes trabalhos [Ferrara et al. 2014, Howard and Kollanyi 2016, Ratkiewicz et al. 2011, Rizoiu et al. 2018]. Em [Rizoiu et al. 2018], os autores investigam como bots interferiram na propagação de tweets durantes os debates nas Eleições Presidenciais nos Estados Unidos em 2016 e os caracterizaram de acordo com sua influência e polaridade política. O fenômeno de astroturfing foi estudado em [Ratkiewicz et al. 2011] com o objetivo de estudar como bots podem afetar a disseminação de informações sobre política nos Estados Unidos. A interação de bots com usuários comuns durante o referendo sobre a permanência do Reino Unido na União Europeia (Brexit) foi analisada em [Howard and Kollanyi 2016], e os autores concluíram que bots exerceram funções estratégicas e interferiram nas discussões online. No trabalho [Abokhodair et al. 2015] os autores revelaram uma rede de bots relacionada à Guerra Civil na Síria e calcularam a influência e a interação de bots com usuários comuns.

A Eleição Presidencial do Estados Unidos em 2016 foi um evento político de grande interesse acadêmico e tópico de diversas pesquisas. Em [Caetano et al. 2018], os autores desenvolveram um framework para identificar o comportamento de usuários durante a campanha eleitoral e os classificou em diferentes grupos em relação ao engajamento. Os pesquisadores em [Bence Kollanyi and Woolley 2016] identificaram bots e concluíram que tais usuários geraram um grande volume de dados. Já as conclusões obtidas em [Bessi and Ferrara 2016] expõe evidências de que bots podem ter influenciado o processo democrático nas eleições por potencialmente terem afetado a opinião pública por ocuparem posições centrais na rede de difusão de informações no Twitter. Nesse estudo, os autores estimaram que $15 \%$ dos usuários ativos eram bots e que eles foram autores por 
$19 \%$ do conteúdo gerado.

Em [Ferrara et al. 2014] os autores descrevem as possíveis consequências da existência de bots em redes sociais, como afetar o processo democrático alterando a percepção de usuários comuns. Os autores ainda identificaram que bots podem exercer influência significativa em usuários comuns e alertam para a dificuldade de estimar a população de bots na rede e, também, de identificar a interferência na propagação de conteúdo.

\subsection{Métricas de influência}

Nesta seção pesquisamos como medir métricas de influência no Twitter e identificamos que não existe um consenso, mas detectamos a tendência de relacionar influência com métricas de retweet.

O número de retweets como métrica de influência foi avaliado em [Anger and Kittl 2011, Ferrara et al. 2014, Rizoiu et al. 2018], porém em [Anger and Kittl 2011] os autores desenvolveram uma métrica que considera o número de menções a outros usuários e em [Rizoiu et al. 2018] foi calculada a probabilidade de um usuário ser retweetado. Em [Cha et al. 2010, Dubois and Gaffney 2014] os autores consideraram a centralidade dos usuários na rede de seguidores, mas o estudo em [Cha et al. 2010] concluiu que a popularidade de um usuário não está fortemente relacionada com suas métricas de propagação de conteúdo. Nos estudos [Stieglitz and Dang-Xuan 2012, Tinati et al. 2012], os autores geraram uma rede de retweet e estruturaram os perfis de comunicação de usuários por meio de suas métricas de retweet.

Os pesquisadores em [Abokhodair et al. 2015, Bakshy et al. 2011, Bessi and Ferrara 2016, Ratkiewicz et al. 2011] geraram grafos com conexões de retweets para estimar a influência de usuários. A interação entre humanos e bots foi descrita em [Bessi and Ferrara 2016], onde os autores concluíram que os bots têm sucesso em participar da rede de difusão de informações. Em [Abokhodair et al. 2015] os autores evidenciaram que uma rede de bots conseguiu acumular engajamento de usuários comuns e ressaltaram o crescimento dessa rede ao longo do tempo. O trabalho apresentado em [Bakshy et al. 2011] cita métricas de retweet e homofilia como consequência da influência de um usuário.

Os trabalhos [Anger and Kittl 2011, Messias et al. 2013] utilizam de APIs, como Klout e Twitalyzer, para calcular a influência de usuários. Essas APIs geram um medida de influência levando em consideração características do perfil dos usuários e, também, estimam o provável alcance do conteúdo gerado por uma conta.

É possível concluir pela análise dos trabalhos relacionados que bots podem afetar o processo democrático e, portanto, a detecção da presença de bots durante as Eleições Presidenciais no Brasil em 2018 [Cristina Tardáguila and Ortellado 2018, Long 2018, Owen 2018] eleva a necessidade de estudar a interação de humanos e bots nesse contexto.

O objetivo deste trabalho, portanto, é estudar o comportamento de bots a partir da detecção de contas automatizadas no Twitter e por meio do cálculo e comparação de métricas de comportamento. Além disso, avaliamos se tais usuários influenciaram usuários comuns no contexto sociopolítico, utilizando como estudo de caso um evento 
apontado por grande parte da mídia brasileira e internacional, a eleição brasileira para presidente de 2018.

\section{Metodologia}

A metodologia utilizada neste trabalho é composta por duas etapas: A primeira se refere a coleta de dados. Já a segunda, refere-se ao cálculo de métricas dos usuários para detectar bots e para análise de influência exercida na rede. As próximas subseções detalham cada uma destas etapas.

\subsection{Coleta de dados}

A coleta de dados transcorreu-se de 11 de Setembro de 2018 a 5 de Novembro de 2018 com a utilização da Twitter Stream API [Twitter 2018]. Como palavras-chave para busca, utilizamos os nomes dos candidatos à Presidência da República conforme descrito na Tabela 1.

Tabela 1. Palavras-chave utilizadas para coleta de dados

\begin{tabular}{|l|}
\hline Alvaro Dias \\
\hline Cabo Daciolo, Daciolo \\
\hline Ciro Gomes, Ciro \\
\hline Eymael \\
\hline Geraldo Alckmin, Alckmin \\
\hline Guilherme Boulos, Boulos \\
\hline Henrique Meirelles, Meirelles \\
\hline Jair Bolsonaro, Bolsonaro \\
\hline Joao Amoedo, Amoedo, Amoedo \\
\hline Joao Goulart Filho, Joao Goulart \\
\hline Luiz Inácio Lula Da Silva, Lula \\
\hline Fernando Haddad, Haddad \\
\hline Marina Silva, Marina \\
\hline Vera Lucia \\
\hline
\end{tabular}

Como resultado da coleta, obtivemos mais de 300GB de dados contendo cerca de 40 milhões de tweets. Para examinar os dados, restringimos o período de análise do sétimo dia anterior ao quinto 5 posterior ao primeiro e segundo turno das eleições sendo, portanto, 26 dias sob avaliação. Este conjunto de dados compreende-se em 15 milhões de tweets de mais de 2.3 milhões de usuários.

\subsection{Cálculo de métricas de usuários}

Após a coleta de dados, o passo seguinte foi a detecção de bots na base de dados para posterior caracterização e comparação com usuários comuns. Pra tanto, decidimos utiliza Botometer API [Dong and Liu 2018], ferramenta comumente utilizada na tarefa de identificação de bots no Twitter.

A Botometer API recebe como entrada o identificador de um usuário e retorna o valor do CAP (do Inglês Complete Automation Probability, Probabilidade de Automação Completa). O CAP é um valor variável de 0 a 1 que estima a probabilidade de uma conta 
de usuário do Twitter ser completamente automatizada, um bot. O valor de referencia para considerar um usuário como bot é de 0.5 . Sendo assim, consideramos usuários com CAP menor que 0.5 como usuários comuns e com valor maior que 0.5 como usuários bot. Em alguns casos, não foi possível identificar o CAP de usuários e, após uma verificação manual, reunimos esses usuários em um grupo com contas suspensas.

O período de análise de 26 dias agrupou tweets de 2.3 milhões de usuários distintos e, dada a relativa dificuldade em classificar todos eles, decidimos por selecionar os 20 mil usuários mais ativos (em número de tweets publicados) por dia. Com isso, foi possível calcular o CAP de 107,877 usuários. O número de usuários classificados como comuns, bots ou suspensos encontra-se na Tabela 2.

Tabela 2. Classificação de usuários por grupo

\begin{tabular}{|c|c|c|c|c|}
\cline { 2 - 5 } \multicolumn{1}{c|}{} & Comum & Bot & Suspenso & Total \\
\hline Quantidade & 101,254 & 2,031 & 4,602 & 107,887 \\
\hline Percentual & $93.85 \%$ & $1.88 \%$ & $4.27 \%$ & $100.00 \%$ \\
\hline
\end{tabular}

O trabalho que apresenta a Botometer API [Dong and Liu 2018] descreve as métricas mais estatisticamente importantes para o cálculo de automação de usuários do Twitter. Com isso, nos inspiramos nessas métricas para selecionar e calcular outras 18 métricas para cada um dos usuários classificados a fim de comparar o comportamento de bots com usuários comuns. Em seguida, calculamos o valor médio de cada métrica com um nível de confiança de 99\%. Os resultados estão apresentados na Tabela 3.

Tabela 3. Valores médios de métricas de atividade por grupo de usuários

\begin{tabular}{|l|r|r|r|}
\hline Métrica & Comum & Bot & Suspenso \\
\hline Amigos & $1,148 \pm 34$ & $604 \pm 98$ & $784 \pm 65$ \\
\hline CAP & $0.029 \pm 0.001$ & $0.662 \pm 0.006$ & \\
\hline Contagem de retweets recebidos & $104.79 \pm 18.98$ & $10.18 \pm 7.49$ & $39.32 \pm 25.43$ \\
\hline Favourites ${ }^{\dagger}$ & $14,458 \pm 224$ & $1,029 \pm 375$ & $12,858 \pm 832$ \\
\hline Hashtags distintas publicadas & $9.91 \pm 0.16$ & $6.48 \pm 0.89$ & $7.39 \pm 0.53$ \\
\hline Idade da conta & $1,727 \pm 10$ & $1,352 \pm 70$ & $1,536 \pm 48$ \\
\hline Menções & $20.34 \pm 0.43$ & $7.79 \pm 1.76$ & $21.04 \pm 2.79$ \\
\hline Retweets & $135 \pm 2$ & $64 \pm 12$ & $92 \pm 9$ \\
\hline Retweets de bots & $0.96 \pm 0.17$ & $0.65 \pm 0.85$ & $0.35 \pm 0.19$ \\
\hline Retweets de comuns & $100 \pm 18$ & $9 \pm 6$ & $36 \pm 23$ \\
\hline Retweets de suspensos & $3.18 \pm 0.51$ & $0.32 \pm 0.18$ & $2.90 \pm 1.82$ \\
\hline Statuses (tweets) ${ }^{\dagger}$ & $26,000 \pm 425$ & $5,951 \pm 1,277$ & $26,555 \pm 1,891$ \\
\hline Statuses (tweets) por dia ${ }^{\dagger}$ & $17.47 \pm 0.23$ & $6.19 \pm 1.20$ & $18.83 \pm 1.12$ \\
\hline Seguidores & $3,182 \pm 724$ & $447 \pm 145$ & $1,216 \pm 514$ \\
\hline Taxa de retweet & $0.75 \pm 0.002$ & $0.71 \pm 0.024$ & $0.69 \pm 0.012$ \\
\hline Tweets & $156 \pm 3$ & $79 \pm 12$ & $118 \pm 10$ \\
\hline Tweets com link & $135 \pm 2$ & $70 \pm 11$ & $99 \pm 9$ \\
\hline Usuários distintos de retweet & $65.74 \pm 0.97$ & $38.96 \pm 5.57$ & $52.77 \pm 3.75$ \\
\hline
\end{tabular}

$\dagger$ Valor da métrica se refere à atividade externa ao período de coleta

A literatura atual descreve múltiplas maneiras de se calcular a influência de 
usuários no Twitter: de métricas simples, como contagem de favourites, a mais complexas, como avaliar a centralidade na rede, ou mesmo ferramentas dedicadas. Neste estudo utilizamos duas métricas para avaliar a influência de usuários: número de retweets e centralidade de usuários na rede de rewteets.

O número de retweets é uma métrica que quantifica o número de vezes que um usuário foi retweetado por outro no período em análise. O trabalho [Cha et al. 2010] demonstrou que tal métrica tem relação direta com o engajamento e o alcance do conteúdo gerado por um usuário e, portanto, indica influência. Os valores na Tabela 3 para o número de retweets revelam que o usuário comum médio é o tipo mais influente na rede, seguido pelo usuário suspenso e pelos bots. Essa métrica, porém, não atesta se bots foram eficientes em influenciar usuários comuns.

Para tanto, foi necessário determinar se bots foram retweetados por usuários comuns. A métrica denominada retweets de comuns na Tabela 3 na coluna Bot indica o valor médio de retweets de usuários comuns recebidos por bots. O valor obtido $(9 \pm 6)$ indica que um bot médio foi capaz de influenciar usuários comuns.

A segunda métrica de influência é a centralidade de bots no grafo gerado pela conexão de retweets, em que cada usuário é representado por um nó e as arestas são não direcionais. Para calculá-la, utilizamos a metodologia descrita em [Bessi and Ferrara 2016] que aplica o algoritmo $k$-core. Tal algoritmo funciona com a obtenção de subgrafos de outro grafos com a seleção de nós com um grau (k) mínimo. Intuitivamente, é possível considerar que usuários mais influentes ocupam posições mais centrais na rede. Sendo assim, obtivemos os subgrafos para valores crescentes de $\mathrm{k}$ e calculamos o percentual de usuários comuns, bots e suspensos em cada um deles. Com esses resultados, incluímos também na análise os usuários com classificação desconhecida.

\section{Resultados experimentais}

Esta seção de resultados experimentais se divide em duas subseções. Primeiro, se refere a comparação de comportamento de bots com usuários comuns e, segundo, analisamos a influência exercida por bots na rede.

\subsection{Comparação de comportamento de bots e usuários comuns}

Os resultados apresentados na Tabela 2 indicam a presença de cerce 2 mil usuários automatizados no Twitter em meio as discussões sobre os candidatos à Presidência da República. Esse resultado ressalta a necessidade de investigar o comportamento de tais usuários e sua interação com usuários comuns durante as Eleições Presidenciais no Brasil em 2018.

Os resultados na Tabela 3 indicam que, quando comparado com um usuário comum médio, um usuário bot médio:

- Possuem contas mais novas

- Interagem com menos hashtags

- Retweetam menos usuários distintos

- Favouritam menos tweets

- Possuem menos seguidores e menos amigos

- Mencionam menos outros usuários 
- Retweetam menos, mas em proporção parecida

- São menos retweetados

- Publicam menos tweets

- Publicam menos tweets com link

A análise das métricas de atividade dos usuários suspensos permite observar resultados ambíguos. Os valores das métricas de número de favourites, menções, statuses e statuses por dia são mais próximas aos resultados de usuários comuns do que bots. Porém, os valores de hashtags distintas publicadas, número de amigos e número de retweets se aproximam mais aos de usuários bots.

A existência dessa ambiguidade sugere a presença de bots classificados como usuários suspensos. Na tentativa de identificá-los, treinamos um modelo de classificação utilizando o algoritmo Floresta Aleatória. Para treinar o modelo, consideramos 17 métricas (desconsideramos o CAP) listadas na Tabela 3. O modelo gerado apresentou uma acurácia de $87 \%$ e F1 Score de 0.78 , resultando na classificação de 507 bots dentre os 4,602 usuários com contas suspensas. Esse resultado fortalece a suspeita da presença de bots dentre os usuários classificados como suspensos.

Os valores médios para as métricas de atividade são úteis para comparação mas não representam a distribuição dos valores para cada grupo de usuários. Para tanto, geramos os gráficos de CDF (do Inglês Cumulative Distribution Function, Função Distribuição Acumulada) para quatro das métricas apresentadas na Tabela 3: idade da conta, CAP, número de favourites e taxa de retweet. Os gŕficos gerados estao apresentados nas Figuras 1, 2, 3 e 4 a seguir:

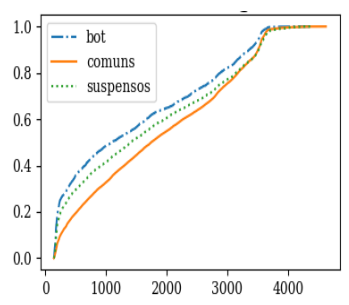

Figura 1. Idade

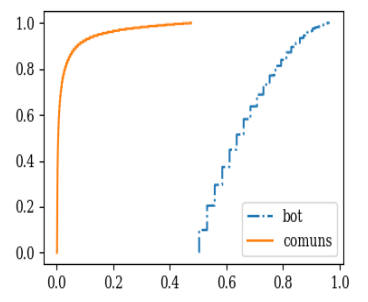

Figura 2. CAP

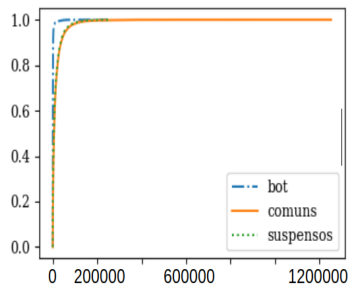

Figura 3. Favourites

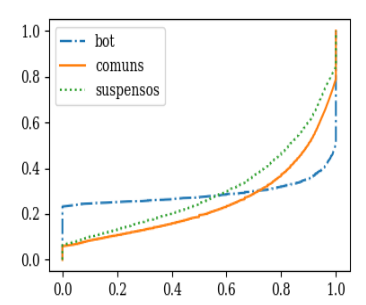

Figura 4. Taxa de retweet

Na Figura 1, observa-se que os bots são contas de usuários mais recentes do que usuários comuns. A CDF para o CAP na Figura 2 ilustra que grande parte dos usuários comuns apresentam valores próximos a 0 enquanto que que para bots os valores estão melhor distribuídos na faixa entre 0.5 e 1 . A Figura 3 indica que bots possuem menos interações por favourites do que usuários comuns. Já a Figura 4 mostra que a taxa de retweet (contagem de retweets divido pelo número de tweets) obtida para os usuários bots concentra-se perto dos valores de 0 e de 1 , indicando uma distribuição bimodal.

\subsection{Influência exercida por bots}

Os resultados obtidos para as métricas de influência de bots estão apresentados nas Figuras 5,6 e 7 a seguir.

A Figura 5 apresenta o resultado do percentual de cada classificação de usuários em relação ao subgrafos gerados do grafo de retweets. Os resultados mostram que com 


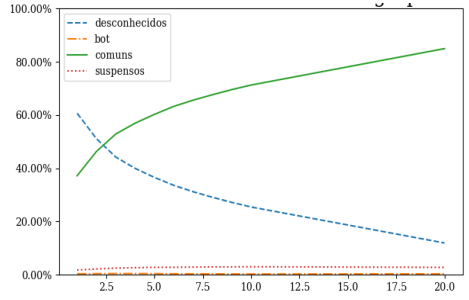

Figura 5. Distribuição de usuários por $k$-core

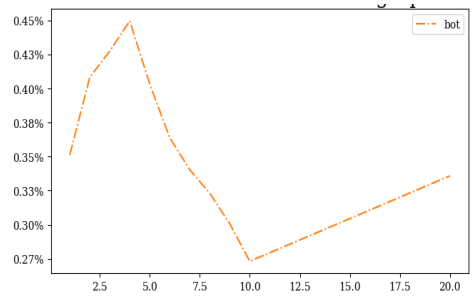

Figura 6. Distribuição de bots por $k$-core

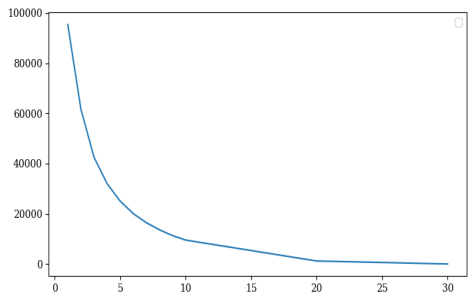

Figura 7. Número de usuários por $k$-core

o crescimento de $\mathrm{k}$ o número de usuários com classificação desconhecida diminui e o número de usuários com classificação conhecida aumenta e indicam, portanto, que os usuários mais influentes foram classificados.

A Figura 6 detalha o percentual de bots nos subgrafos com o crescimento de k. Os resultados indicam que os bots se mantiveram presentes nos subgrafos gerados, ou seja, alguns deles possuíam grande centralidade na rede. Sendo assim, é possível considerar que esses resultados fortalecem os indícios de que usuários automatizados influenciaram usuários comuns, dado que bots ocuparam posições de influência no grafo gerado pela rede de retweets.

A Figura 7 exibe a quantidade de usuários por subgrafos gerados. Esse resultado revela que o valor diminui exponencialmente com o aumento de $\mathrm{k}$, ocorrendo grande restrição de usuários quando subgrafos são selecionados sequencialmente.

\section{Discussão dos resultados}

Os resultados experimentais foram obtidos a fim de responder as Questões de Pequisa definidas. Para tanto, foram coletados cerca de 40 milhões de tweets com referência aos candidatos à Presidência da República nas Eleições no Brasil de 2018, identificamos usuários bots e comparamos sua atividade com usuários comuns. Além disso, calculamos duas métricas para avaliar a influencia de bots sobre usuários comuns.

A QP1 se refere a detecção de bots nas discussões no Twitter sobre as Eleições Presidenciais. Conforme descrito na seção de Resultados Experimentais, utilizamos a Botometer API [Dong and Liu 2018] para classificar mais de 100 mil contas do Twitter e detectamos a presença de cerca de 2 mil bots. Tal resultado indica, portanto, que os bots estavam presentes nas discussões online. Além disso detectamos cerca de 4,5 mil usuários com contas suspensas e, ao gerar um modelo de classificação com o algoritmo de Floresta Aleatória, identificamos outros 507 usuários suspeitos como bots. Esse resultado sugere que o Twitter suspendeu alguns dos usuários bots.

A CDF para o valor do CAP, disponível na Figura 2, mostra que cerca de $80 \%$ dos usuários comuns possuem valores pequenos, ou seja, a maior parte deles demonstra um comportamento humano. O 20\% restante obteve CAP crescente, sugerindo que podem ser usuários com baixo nível de automação. Essa mesma CDF revela valores diversos de CAP para os bots, sinalizando que tais usuários possuem comportamento diverso na rede.

Na QP1 questiona-se a comparação do comportamento de bots e usuários comuns. Para tanto, calculamos o intervalo de confiança de 18 métricas de atividade para cada uma das classificações de usuários e os apresentamos na Tabela 3. Os resultados indicam 
diferenças nas métricas atividade e, portanto, do comportamento entre bots e usuários comuns. Por exemplo, é possível observar que os bots entraram mais recentemente na rede que usuários comuns (também observável na Figura 1), são menos ativos (métricas de tweets e retweets) e tem menos engajamento de outros usuários (métricas de amigos, seguidores e retweets recebidos). Além disso, a Figura 4 indica um comportamento bimodal na distribuição da métrica de taxa de retweets, não observável em usuário comuns. Esse resultado sugere a existência de três grupos de bots: os que geram conteúdo, os que promovem conteúdo por meio de e retweets e, em menor número, os que exercem as duas atividades.

É observável também que, em comparação ao usuário comum médio, um bot médio publica menos hashtags distintas, retweeta menos usuários e tem menos favourites. Tais resultados são indícios de que os bots tem menos engajamento na rede do que usuários comuns e, além disso, tem dificuldade em seguir conteúdos e usuários diversificados.

Para avaliar se bots e usuários comuns se interessaram pelos mesmos assuntos analisamos as hashtags compartilhadas. Para tanto, criamos dois rankings de hashtags compartilhadas, um para bots e um para usuários comuns, e calculamos a relação entre eles utilizando o coeficiente de correlação de Spearman e obtivemos $\rho$ igual a 0.20. Ainda detectamos que, dentre as 5 mil hashtags mais publicadas pelos bots, 654 não estavam presentes nas 5 mil mais publicadas por usuários comuns. Esses resultados indicam uma fraca correlação entre os dois rankings mas indica que os dois grupos de usuários estavam interessados nos mesmos tópicos.

Na QP2 questiona-se o objetivo é determinar se bots influenciaram usuários comuns. Para tanto, calculamos métricas de influência e detectamos que usuários comuns retweetaram bots e que usuários automatizados ocuparam posições centrais na rede (como visto na Figura 6). Esses resultados indicam que bots influenciaram usuários comuns. De forma geral, os resultados indicam que os bots nesse período mantiveram baixa atividade de publicação e engajamento (se comparados com usuários comuns) mas conseguiram se manter ativos na rede social e ainda influenciar outros usuários.

Os resultados obtidos são indícios da influência contas automatizadas nas discussões online no Twitter durante as Eleições Presidenciais no Brasil em 2018. De fato, tal constatação tem forte relação com outros trabalhos recentes que evidenciam a utilização de bots para influenciar discussões online por motivos políticos [Abokhodair et al. 2015, Bessi and Ferrara 2016]. Com isso, os resultados neste trabalho podem ser utilizados como evidência e, também, incitar outros estudos sobre a detecção de bots e as possíveis consequências na sociedade. Nesse sentido, estudos [Abokhodair et al. 2015, Bessi and Ferrara 2016, Ferrara et al. 2014, Rizoiu et al. 2018] que descrevem consequências negativas para a sociedade e para o processo democrático. Sendo assim, o conhecimento desse contexto deve ser amplamente estudado e divulgado, a fim de evitar tais efeitos danosos à sociedade.

\section{Conclusão}

Os objetivos deste trabalho são detectar a presença de bots no Twitter durante as Eleições Presidenciais no Brasil em 2018, comparar o comportamento deles com usuários comuns e estimar se eles influenciaram outros. Concluímos que cerca de $2 \%$ dos usuários mais 
ativos no período de análise indicavam alto nível de automação e que eles foram eficientes em influenciar usuários comuns. Sendo assim, os resultados obtidos realçam a necessidade de investimento na detecção de bots em redes sociais e, também, no estudos dos impactos causado por bots no mundo real.

Em trabalhos futuros pretendemos usar informações temporais baseadas no comportamento dos bots, contribuindo para a detecção desses perfis automatizados. Além disso, esperamos estudar a participação de usuários automatizados no compartilhamento de conteúdo no Twitter.

\section{Referências}

Abokhodair, N., Yoo, D., and McDonald, D. W. (2015). Dissecting a social botnet: Growth, content and influence in twitter. In Proceedings of the 18th ACM Conference on Computer Supported Cooperative Work \& Social Computing, CSCW 2015, Vancouver, BC, Canada, March 14 - 18, 2015, pages 839-851.

Anger, I. and Kittl, C. (2011). Measuring influence on twitter. In I-KNOW 2011, 11th International Conference on Knowledge Management and Knowledge Technologies, Graz, Austria, September 7-9, 2011, page 31.

Bakshy, E., Hofman, J. M., Mason, W. A., and Watts, D. J. (2011). Everyone's an influencer: quantifying influence on twitter. In Proceedings of the Forth International Conference on Web Search and Web Data Mining, WSDM 2011, Hong Kong, China, February 9-12, 2011, pages 65-74.

Bence Kollanyi, P. N. H. and Woolley, S. C. (2016). Bots and automation over twitter during the u.s. election.

Bessi, A. and Ferrara, E. (2016). Social bots distort the 2016 U.S. presidential election online discussion. First Monday, 21(11).

Caetano, J. A., Almeida, J. M., and Marques-Neto, H. T. (2018). Characterizing politically engaged users' behavior during the 2016 US presidential campaign. In Brandes, U., Reddy, C., and Tagarelli, A., editors, IEEE/ACM 2018 International Conference on Advances in Social Networks Analysis and Mining, ASONAM 2018, Barcelona, Spain, August 28-31, 2018, pages 523-530. IEEE Computer Society.

Caetano, J. A., Lima, H. S., dos Santos, M. F., and Marques-Neto, H. T. (2017). Utilizando análise de sentimentos para definição da homofilia política dos usuários do twitter durante a eleição presidencial americana de 2016.

Cha, M., Haddadi, H., Benevenuto, F., and Gummadi, P. K. (2010). Measuring user influence in twitter: The million follower fallacy. In Proceedings of the Fourth International Conference on Weblogs and Social Media, ICWSM 2010, Washington, DC, USA, May 23-26, 2010.

Cristina Tardáguila, F. B. and Ortellado, P. (2018). Fake news is poisoning brazilian politics. whatsapp can stop it. https://www. nytimes.com/2018/10/ 17/opinion/brazil-election-fake-news-whatsapp.html. Accessed: 2018-12-02.

Dickerson, J. P., Kagan, V., and Subrahmanian, V. S. (2014). Using sentiment to detect bots on twitter: Are humans more opinionated than bots? In 2014 IEEE/ACM Inter- 
national Conference on Advances in Social Networks Analysis and Mining, ASONAM 2014, Beijing, China, August 17-20, 2014, pages 620-627.

Dong, G. and Liu, H. (2018). Feature engineering for machine learning and data analytics.

Dubois, E. and Gaffney, D. (2014). The multiple facets of influence: Identifying political influentials and opinion leaders on twitter. American Behavioral Scientist, 58(10) 1260.

Ferrara, E., Varol, O., Davis, C. A., Menczer, F., and Flammini, A. (2014). The rise of social bots. CoRR, abs/1407.5225.

Howard, P. N. and Kollanyi, B. (2016). Bots, \#strongerin, and \#brexit: Computational propaganda during the UK-EU referendum. CoRR, abs/1606.06356.

Long, C. (2018). How false news haunted the brazilian elections. https://slate.com/technology/2018/10/ brazil-election-fake-news-whatsapp-facebook.html. Accessed: 2018-12-02.

Messias, J., Schmidt, L., Oliveira, R., and Benevenuto, F. (2013). You followed my bot! transforming robots into influential users in twitter. First Monday, 18(7).

Owen, L. H. (2018). What to know about whatsapp in brazil ahead of sunday's election. http://www.niemanlab.org/2018/10/ what-to-know-about-whatsapp-in-brazil-ahead-of-sundays-election/. Accessed: 2018-12-02.

Ratkiewicz, J., Conover, M. D., Meiss, M. R., Goncalves, B., Flammini, A., and Menczer, F. (2011). Detecting and tracking political abuse in social media. In Adamic, L. A., Baeza-Yates, R. A., and Counts, S., editors, Proceedings of the Fifth International Conference on Weblogs and Social Media, Barcelona, Catalonia, Spain, July 17-21, 2011. The AAAI Press.

Rizoiu, M., Graham, T., Zhang, R., Zhang, Y., Ackland, R., and Xie, L. (2018). \#debatenight: The role and influence of socialbots on twitter during the 1st 2016 U.S. presidential debate. In Proceedings of the Twelfth International Conference on Web and Social Media, ICWSM 2018, Stanford, California, USA, June 25-28, 2018., pages 300-309.

Stieglitz, S. and Dang-Xuan, L. (2012). Political communication and influence through microblogging-an empirical analysis of sentiment in twitter messages and retweet behavior. In 45th Hawaii International International Conference on Systems Science (HICSS-45 2012), Proceedings, 4-7 January 2012, Grand Wailea, Maui, HI, USA, pages 3500-3509. IEEE Computer Society.

Tinati, R., Carr, L., Hall, W., and Bentwood, J. (2012). Identifying communicator roles in twitter. In Mille, A., Gandon, F. L., Misselis, J., Rabinovich, M., and Staab, S., editors, Proceedings of the 21st World Wide Web Conference, WWW 2012, Lyon, France, April 16-20, 2012 (Companion Volume), pages 1161-1168. ACM.

Twitter (2018). Twitter developper. https://developer.twitter.com/en. html. Accessed: 2018-12-02. 